\title{
Recent Advances in the Direct Nucleophilic Substitution of Allylic Alcohols through $S_{\mathrm{N}} 1$-Type Reactions
}

\author{
Alejandro Baeza, ${ }^{*}$ Carmen Nájera* \\ Departamento de Química Orgánica and Instituto de Síntesis Orgánica, University of Alicante, Apdo.99, 03080 Alicante, Spain \\ Fax+34(965)903549; E-mail: alex.baeza@ua.es; E-mail: cnajera@ua.es \\ Received: 03.10.2013; Accepted after revision: 06.11.2013
}

\begin{abstract}
Direct nucleophilic substitution reactions of allylic alcohols are environmentally friendly, since they generate only water as a byproduct, allowing access to new allylic compounds. This reaction has, thus, attracted the interest of the chemical community and several strategies have been developed for its successful accomplishment. This review gathers the latest advances in this methodology involving $\mathrm{S}_{\mathrm{N}} 1$-type reactions.

1 Introduction

$2 \quad \mathrm{~S}_{\mathrm{N}}$ 1-Type Direct Nucleophilic Substitution Reactions of Allylic Alcohols

2.1 Lewis Acids as Catalysts

2.2 Brønsted Acids as Catalysts

2.3 Other Promoters

3 Conclusions and Outlook
\end{abstract}

Key words: $\mathrm{S}_{\mathrm{N}} 1$ reaction, allylic substitution, carbocations, allylic alcohols, green chemistry

\section{$1 \quad$ Introduction}

The need to save resources and concerns about our environment have led to the development of new strategies in organic synthesis that are able to fulfill these requirements. ${ }^{1}$ Thus, new environmentally friendly processes have been established that minimize waste production by applying the atom-economy concept ${ }^{2}$ and avoiding the use of toxic or expensive reagents and/or catalysts.

The allylic substitution reaction is a widespread methodology that is successfully employed in organic synthesis to obtain new allylic compounds. ${ }^{3}$ Traditionally, this process employs alcohol derivatives, such as allylic carbonates, acetates, phosphates, and halides as substrates, and this generates a stoichiometric amount of waste in their preparation and on their reaction with the corresponding nucleophile. Therefore, a more attractive strategy from both a practical and environmentally point of view would be the direct use of allylic alcohols in this transformation, since they are readily available compounds and water would be the only byproduct formed (Scheme 1). ${ }^{1}$

SYNTHESIS 2014, 46, 0025-0034

Advanced online publication: 25.11.2013

DOI: 10.1055/s-0033-1340316; Art ID: SS-2013-M0658-SR

(C) Georg Thieme Verlag Stuttgart · New York

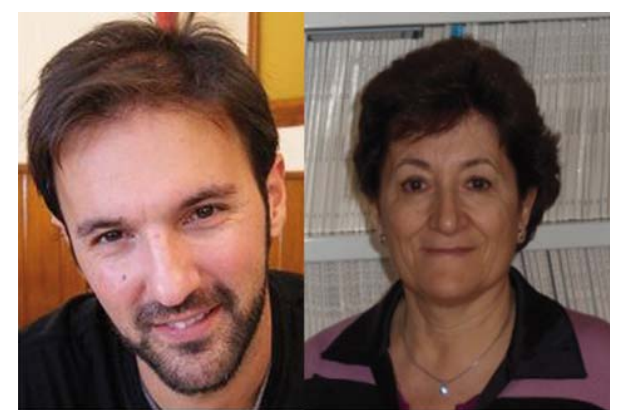

Alejandro Baeza was born in Alicante (Spain) in 1979. He studied chemistry at the University of Alicante and he received his M.Sc. (2003) and $\mathrm{Ph}$. D. degrees (2006) from here under the supervision of Prof. José Miguel Sansano and Prof. Carmen Nájera. He was a postdoctoral researcher in Prof. Pfaltz's research group (2007-2010). In 2010 he returned to Alicante and joined the research group of Prof. Carmen Nájera. His main research interests focus on the development of new environmentally friendly methodologies, especially in asymmetric synthesis. Carmen Nájera was born in Nájera (La Rioja). She graduated from the University of Zaragoza in 1973, and obtained her doctorate in chemistry from the University of Oviedo in 1979. This was followed by postdoctoral positions at ETH (Zurich), Dyson Perrins Laboratory (Oxford), Harvard University, and Uppsala University. She became an Associate Professor in 1985 at the University of Oviedo and a Full Professor in 1993 at the University of Alicante. Prof. Carmen Najera is the co-author of more than 300 papers and book chapters, and she has been a visiting professor at the University of Arizona (Tucson, USA), Universidad Nacional del Sur (Bahia Blanca, Argentina), Université Louis Pasteur (Strasbourg, France), and Ecole Nationale Superiéure de Chimie de Paris (France). She was awarded the 'Organic Chemistry Prize' from Real Sociedad Española de Química (2006), 'Rosalind Franklin International Lectureship' from the Royal Society of Chemistry (2006), and the SCF 2010 French-Spanish Prize from the Société Chimique de France. In 2012 she was named a Member of the Real Academia de Ciencias Exactas, Físicas y Naturales, and she was appointed a Member of the European Academy of Sciences and Arts.

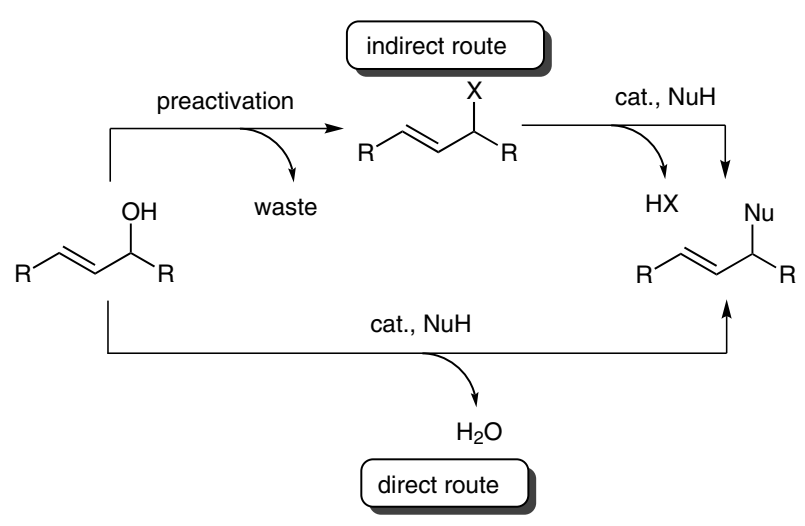

Scheme 1 Direct and indirect routes for allylic substitution reactions 


\section{2}

\section{$S_{\mathrm{N}}$ 1-Type Direct Nucleophilic Substitution Reactions of Allylic Alcohols}

The advantages of free alcohols in allylic substitution reactions have the attention of organic chemists, and numerous procedures have been devised to accomplish this purpose. However, free allylic alcohols have two main drawbacks: the poor ability of the hydroxyl function as a leaving group and the formation of a stoichiometric amount of water; this requires the use of a water-tolerant catalyst able to activate the hydroxy group. Thus, three main strategies, based on the catalysts employed, have been developed to overcome these problems (Scheme 2):

The use of transition-metal-based catalysts (such as Pd, Ir, $\mathrm{Pt}$, Rh etc.) implying a $\pi$-allyl intermediate in a TsujiTrost-type reaction. ${ }^{4}$

The employment of Brønsted or oxophilic Lewis acids forming the corresponding carbocationic intermediate in an $\mathrm{S}_{\mathrm{N}} 1$-type reaction. ${ }^{5}$

The use of soft $\pi$-Lewis acids, such as $\mathrm{Au}(\mathrm{I})$ or $\mathrm{Hg}(\mathrm{II})$, able to activate the double bond, and hence facilitating the nucleophilic attack in an $\mathrm{S}_{\mathrm{N}} 2^{\prime}$-type reaction. ${ }^{6}$

This review discusses the most relevant recent work published since 2011 that uses catalysts or promoters, which implies the formation of a carbocationic intermediate in an $\mathrm{S}_{\mathrm{N}}$ 1-type reaction, for direct allylic substitution reactions using free alcohols. ${ }^{5}$

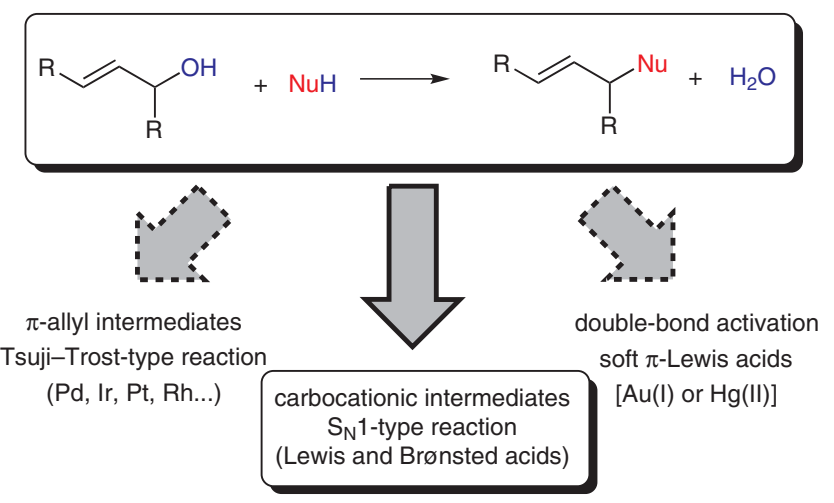

Scheme 2 Strategies to perform allylic substitution reactions of free alcohols

\subsection{Lewis Acids as Catalysts}

The use of oxophilic Lewis acids as catalysts grows rapidly, despite the great progress already achieved. ${ }^{5}$ Generally, the Lewis acids employed tend to be readily available. ${ }^{7}$ As depicted in Scheme 3, the mode of activation of these catalysts consists of the interaction of the Lewis acid (LA) with the hydroxyl group, forming a hydroxo-anionic complex and the allylic carbocation intermediate. After attack of the nucleophile and proton release, the Lewis acidity is re-established by the loss of a molecule of water.

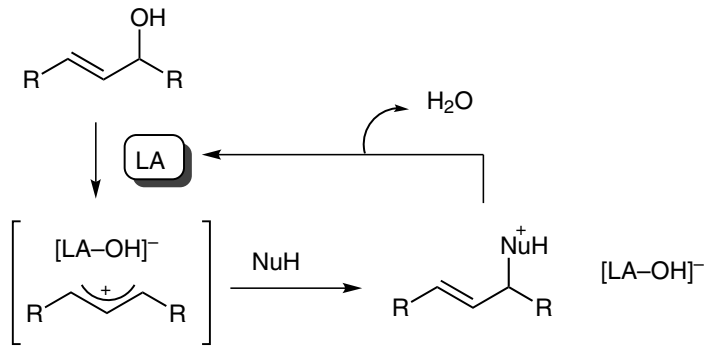

Scheme 3 Activation mode of allylic alcohols by Lewis acids

Our group has reported the use of silver(I) triflate for the direct amination of allylic alcohols; silver salts are readily available Lewis acids that had not previously been used in direct intermolecular allylic substitution reactions using allylic alcohols. ${ }^{8}$ The use of silver(I) triflate in low catalyst loadings (2-5 mol\%) allowed the successful amination of various allylic alcohols with sulfonamides, carbamates, and anilines [Scheme 4, (a)].

The Che group also used silver(I) triflate for the allylation of electron-rich arenes and heteroarenes in a FriedelCrafts-type reaction that achieved good to excellent results [Scheme 4, (b)]. ${ }^{9}$ Che and co-workers also described the use of $\mathrm{Ag}_{3} \mathrm{PW}_{12} \mathrm{O}_{40}$ as a heterogeneous catalyst for this transformation that gives comparable results; $\mathrm{Ag}_{3} \mathrm{PW}_{12} \mathrm{O}_{40}$ was recyclable by simply filtration for up to five cycles. ${ }^{9}$ Rueping and co-workers described the use of silver(I) triflate for the direct azidation of allylic alcohols $\left[\right.$ Scheme 4, (c)] ${ }^{10}$ In all cases the regioselectivity of the process was governed by the stability of the carbocation intermediate.

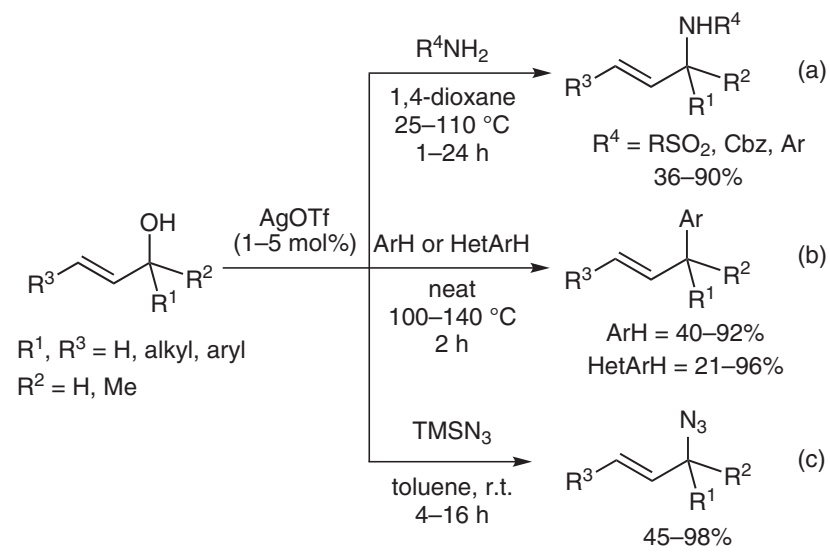

Scheme 4 Nucleophilic substitutions catalyzed by AgOTf

Another late transition metal that had not previously been frequently employed in this type of transformation is copper. ${ }^{5}$ The Wang and Zhang group reported the formation of $\mathrm{C}-\mathrm{C}$ bonds between terminal alkynes and allylic alcohols catalyzed by $\mathrm{Cu}(\mathrm{OTf})_{2}$ with low catalyst loadings $(0.5 \mathrm{~mol} \%)$ and using 1,2-dibromoethane as the solvent. Remarkably, other Lewis and Brønsted acids commonly employed in direct substitution reactions of alcohols failed or, at best, gave poor results for this particular trans- 
formation. This methodology was also applied to benzylic alcohols (Scheme 5). ${ }^{11}$

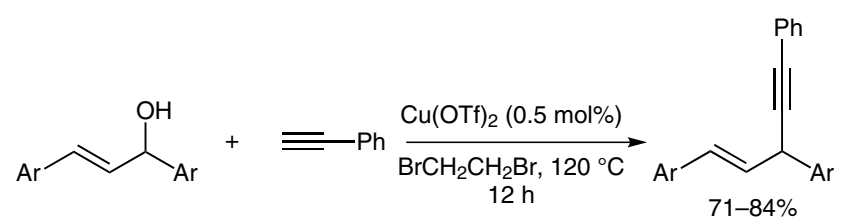

Scheme 5 Alkynylation of allylic alcohols catalyzed by $\mathrm{Cu}(\mathrm{OTf})_{2}$

As discussed, $\mathrm{Au}(\mathrm{I})$ is considered as a $\pi$-philic Lewis acid, and, therefore, a carbocationic intermediate is rarely involved when their complexes are used for this reaction. ${ }^{6,12}$ However, $\mathrm{Au}(\mathrm{III})$ is not the same, as in this higher oxidation state the gold species are considered oxophilic and can activate the hydroxyl group generating a carbocation. ${ }^{5}$ Thus, the Ohshima and Mashima group took advantage of this well-known fact, ${ }^{6}$ and they reported the use of $\mathrm{NaAuCl}_{4} \cdot 2 \mathrm{H}_{2} \mathrm{O}(1 \mathrm{~mol} \%)$ for the direct allylic amination reaction of different linear allylic alcohols using highly functionalized nitrogen nucleophiles (containing other functional groups such as protected alcohols, carbonyl groups, acetals etc.) in refluxing dichloromethane. ${ }^{13}$ This group also described the use of aluminum salts, more precisely $\mathrm{Al}(\mathrm{OTf})_{3}$, which were unexplored as catalysts for this type of transformation. ${ }^{14}$ In this case only $1 \mathrm{~mol} \%$ of catalyst loading in nitromethane as solvent was sufficient to achieve high yields within minutes for most examples described. In both cases, the observed racemization of optically enriched substrates during the substitution process supports an $\mathrm{S}_{\mathrm{N}} 1$-type mechanism. In addition, in the case of $\mathrm{Al}(\mathrm{OTf})_{3}$ the authors also performed the direct amination under microwave irradiation and this shortened the reaction times, especially in the most challenging examples, and maintained the excellent results. ${ }^{14}$

The same racemization in the aminated product when starting from an enantioenriched allylic alcohol was observed by Yamamoto and co-workers for the intermolecular amination of alcohols using sulfamates as nucleophiles and $\mathrm{Hg}(\mathrm{OTf})_{2}$ as catalyst, which, as in the previous gold case, is commonly classified as a soft $\pi$ Lewis acid. This fact, together with double bond isomerizations, points towards an $\mathrm{S}_{\mathrm{N}} 1$-type mechanism. Thus, high yields were obtained at room temperature with only $2 \mathrm{~mol} \%$ catalyst loading. The reaction shows high functional group tolerance, although in some cases higher temperatures were needed. In addition, a supported version of this catalyst was also successfully employed and reused for up to five cycles by simply filtration without apparent metal leaching, for example, in the reaction between cyclohex-2-enol (1) and methyl sulfamate (2) (Scheme 6). ${ }^{15}$

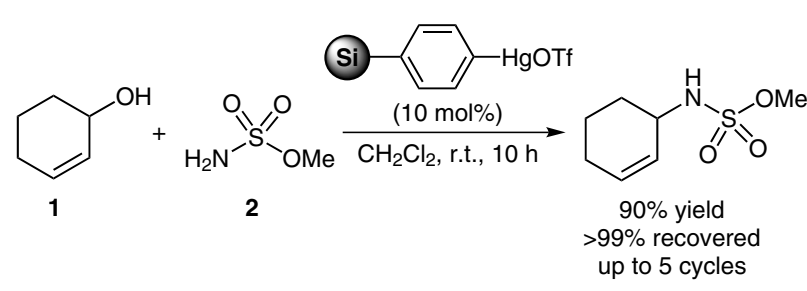

Scheme 6 A supported mercury(II) salt as a recyclable catalyst

Zinc(II)-based salts efficiently catalyze the direct allylic substitution of alcohols employing 1,3-dicarbonyl compounds as nucleophiles under microwave irradiation. ${ }^{5,16}$ An additional attractive application from an environmental point of view has been developed based on the use of $\mathrm{ZnBr}_{2}$ acting as a catalyst for the solvent-free allylic substitution reaction of alcohols, using indoles and nitrogen compounds (mainly sulfonamides and electron-poor anilines) as nucleophiles under high-speed vibration milling conditions. Other common Lewis acids employed in this reaction, such as $\mathrm{FeCl}_{3}, \mathrm{AlCl}_{3}$, and $\mathrm{CuCl}_{2}$, afforded the corresponding products in lower yields under the same conditions. Unfortunately, a relatively high catalyst loading $(20 \mathrm{~mol} \%)$ was necessary to achieve high yields. ${ }^{17}$

In addition, a further two processes use zinc salts in a $\mathrm{C}-$ $\mathrm{C}$ bond-forming reaction. The first uses $15 \mathrm{~mol} \%$ $\mathrm{Zn}(\mathrm{OTf})_{2}$ as the catalyst and TMSCN (4) as the cyanide source for the direct cyanation of benzylic and allylic alcohols, such as (E)-1,3-diphenylprop-2-en-1-ol (3), giving the corresponding nitriles in good yields (Scheme 7). ${ }^{18}$

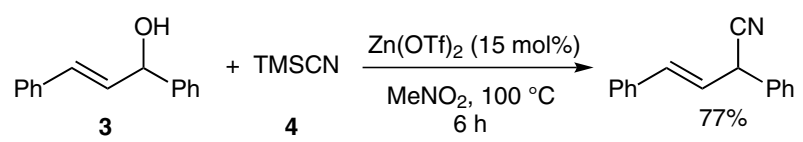

Scheme 7 Cyanation of allylic alcohols catalyzed by zinc triflate

The second process, from the Ma group, uses allenes as nucleophiles in the reaction with in situ formed carbocations derived from allylic alcohols. In this case zinc halides, although used in stoichiometric amounts, were the promoters. Depending on the allene structure and the zinc halide employed, different products were isolated (Scheme 8). Thus, when the R group was alkyl or aryl and $\mathrm{ZnCl}_{2}$ was employed, the corresponding indene derivatives were obtained as a consequence of nucleophilic attack of the allene on the transient carbocation followed by a Friedel-Crafts-type reaction. However, for monosubstituted allenes $(\mathrm{R}=\mathrm{H})$, the corresponding halopenta-1,4-dienes were obtained regardless of the zinc salt employed. ${ }^{19}$ 


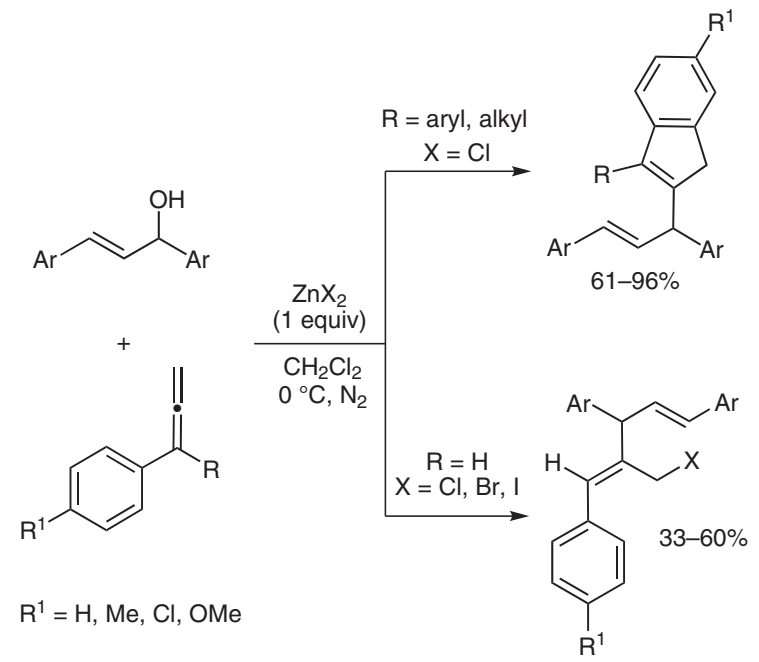

Scheme 8 Allenes as nucleophiles in zinc(II)-mediated reactions with allylic alcohols

Bismuth(III) triflate is an excellent catalyst for the direct allylic substitution of free alcohols. ${ }^{5,20}$ However, a new protocol by the Laali group uses ionic liquids as environmentally friendly and reusable reaction media in combination with $10 \mathrm{~mol} \%$ of $\mathrm{Bi}(\mathrm{OTf})_{3}$ as the catalyst. Under these conditions silyl-based nucleophiles were successfully introduced in short reaction times affording the corresponding products in high yields (Scheme 9). The ionic liquid $[\mathrm{BMIN}]\left[\mathrm{BF}_{4}\right]$ was recovered for up to six cycles without affecting the chemical yield. ${ }^{21 a} \mathrm{~A}$ similar strategy, but using $\mathrm{Bi}\left(\mathrm{NO}_{3}\right)_{3} \cdot 5 \mathrm{H}_{2} \mathrm{O}$ as the catalyst, was followed by the same group when using 1,3-dicarbonyl compounds as nucleophiles. $^{21 b}$

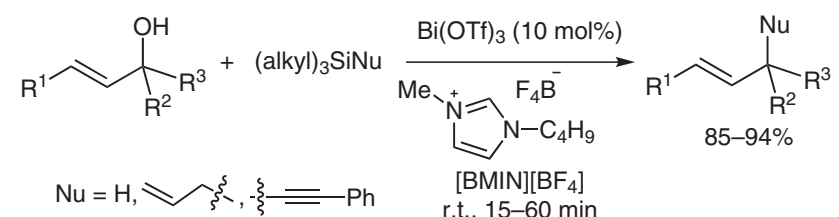

Scheme 9 Bismuth(III)-catalyzed allylic substitution of alcohols in ionic liquids

Other metal species that act as Lewis acids which are able to catalyze the direct allylic substitution of alcohols that had not previously been explored are $\mathrm{SbCl}_{3}$ and $\mathrm{Re}_{2} \mathrm{O}_{7}$. Nayak et al. used $\mathrm{SbCl}_{3}$ as a catalyst for the allylation (and alkylation using benzylic alcohols) of electron-rich arenes and heteroarenes with (E)-1,3-diphenylprop-2-en-1-ol (3) as the substrate in a microwave-accelerated reaction that achieved good yields in short reaction times, although a relatively large amount of catalyst $(15 \mathrm{~mol} \%)$ was needed [Scheme 10, (a)].22 In contrast, $\mathrm{Re}_{2} \mathrm{O}_{7}$ showed excellent activity as a catalyst for the amination of allylic alcohols with different nitrogen-containing nucleophiles; only 1.5 mol\% of $\mathrm{Re}_{2} \mathrm{O}_{7}$ was required at room temperature to reach high yields [Scheme 10, (b)]. ${ }^{23}$

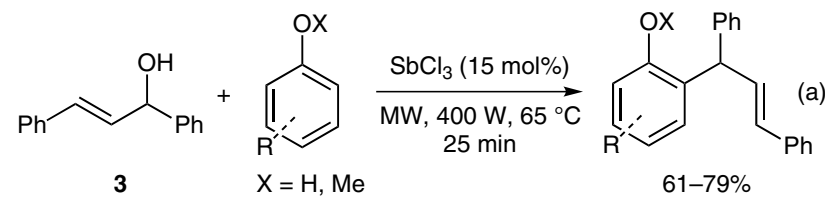<smiles>[R]C(O)(/C=C/[H])[R]([H])([H])/C=C/[3H]</smiles>

$\mathrm{R}^{1}, \mathrm{R}^{2}=\mathrm{H}$, alkyl, aryl $\mathrm{PG}=\mathrm{Cbz}, \mathrm{Boc}, \mathrm{Ts}, \mathrm{Ns} \quad 61-99 \%$

$$
\mathrm{R}^{3}=\mathrm{H}, \mathrm{Me} \quad \mathrm{n}=0,1,2
$$

The alkaline-earth metals have attracted much less attention as Lewis acids that are able to activate the hydroxy group and, hence, promote this transformation. The Niggemann group has studied the use of calcium salts as catalysts for the direct substitution of alcohols in an $\mathrm{S}_{\mathrm{N}} 1-$ type reaction. Concretely, they have successfully utilized calcium triflimide in combination with an ammonium salt for the nucleophilic substitution reactions of various allylic alcohols. Thus, depending on the nucleophile employed the direct amination, Friedel-Crafts-type arylation, and allylation/alkenylation reaction were achieved (Scheme 11). ${ }^{25}$ 


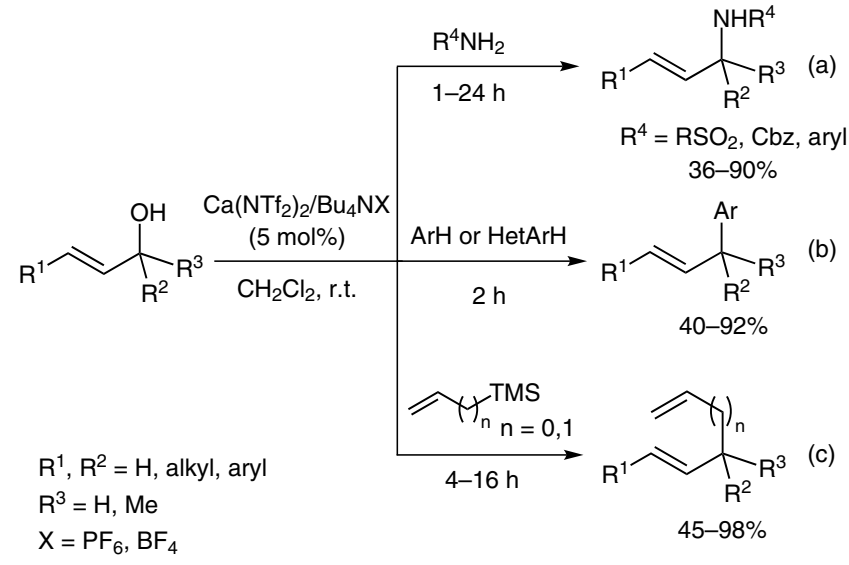

Scheme 11 Calcium triflimide catalyzed allylic substitution with free alcohols

Iodine efficiently catalyzes the direct allylic substitution reaction of alcohols under mild conditions giving satisfactory results with different nucleophiles. ${ }^{5}$ The Liu group took advantage of this fact and applied this methodology to the allylation of a wide variety of nucleophiles, such as arenes, nitrogen nucleophiles, and 1,3-dicarbonyl compounds, achieving excellent results. ${ }^{26}$

The Ji group used the combination of $\mathrm{Fe} / \mathrm{I}_{2} / \mathrm{NaI}$ in stoichiometric amounts for the synthesis of alkenyl iodides in good yields and diastereoselectivities through the reaction of alkynes with allylic and benzylic alcohols (a representative example is shown in Scheme 12). ${ }^{27}$ Although the exact mechanism remains unclear, it is proposed that a carbocation derived from the corresponding alcohol is formed by reaction either with iodine or iron.

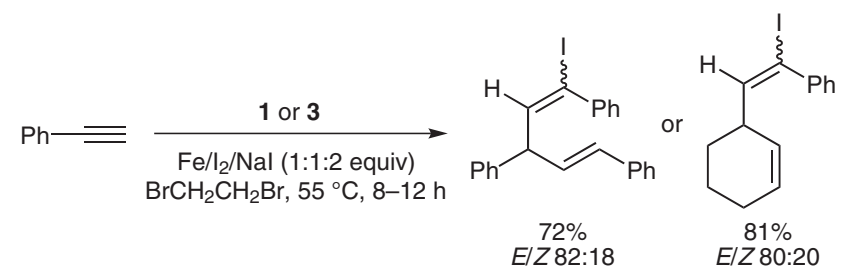

Scheme 12 Iodoalkenylation of allylic alcohols

Iron(III) chloride has already shown its effectiveness as a catalyst for this synthetic transformation in its inter- and intramolecular versions. ${ }^{5,28}$ However, our research group used $\mathrm{FeCl}_{3} \cdot 6 \mathrm{H}_{2} \mathrm{O}$, which, in contrast to anhydrous $\mathrm{FeCl}_{3}$ which had been used previously, is more economic and can be used in an open-air flask using technical grade solvents. Our work focused on direct allylic amination reactions with free alcohols using $\mathrm{FeCl}_{3} \cdot 6 \mathrm{H}_{2} \mathrm{O}$ or TfOH as a representative, readily available Brønsted acid (see Section 2.2 for further details) in a comparative study. ${ }^{29}$ In this way sulfonamides, anilines, carbamates, amides, and even triazoles were allylated, generally in high yields, us- ing a wide range of allylic alcohols as alkylating agents through a $\mathrm{S}_{\mathrm{N}} 1$-type reaction under mild reaction conditions $\left(25-50^{\circ} \mathrm{C}\right)$ and using a relatively low catalyst loading (normally $5-10 \mathrm{~mol} \%$ of $\mathrm{FeCl}_{3} \cdot 6 \mathrm{H}_{2} \mathrm{O}$ ) with dioxane as the solvent. The $\mathrm{S}_{\mathrm{N}} 1$ reaction mechanism was confirmed not only by the detected racemization of enantioenriched allylic $(E)$-1-phenylpent-1-en-3-ol (5) [Scheme 13, (a)] after the reaction took place, but also because the use of isomeric alcohols $\mathbf{6}, 7$, and 8 produced the same amination product [Scheme 13, (b)]. This fact, which governs the regiochemistry of the process, suggest a common carbocationic allylic intermediate that leads to the most stable, from a thermodynamic point of view, double bond prior to the amination. In addition other carbonucleophiles such as silyl-based, aromatics, and malonates were also successfully allylated employing this methodology. ${ }^{29}$

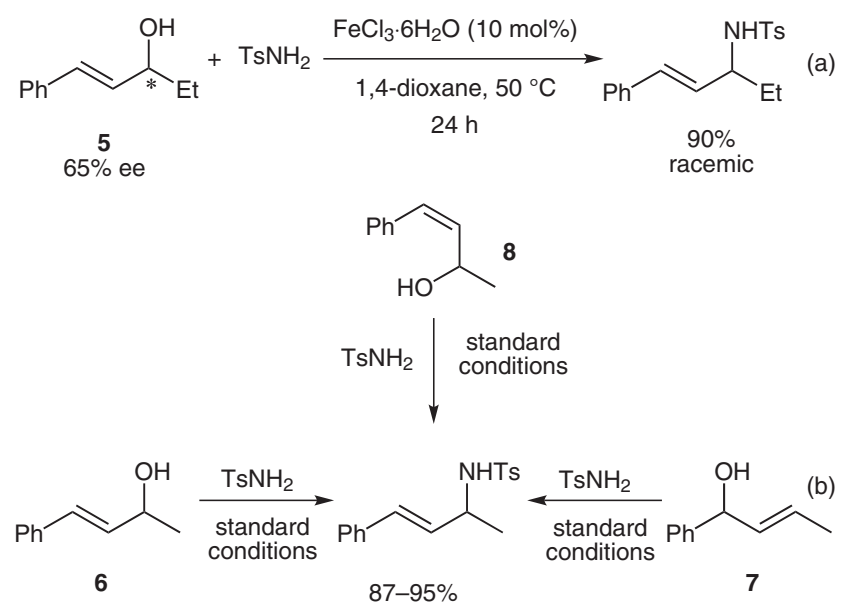

standard conditions: $\mathrm{FeCl}_{3} \cdot 6 \mathrm{H}_{2} \mathrm{O}(5 \mathrm{~mol} \%), 50{ }^{\circ} \mathrm{C}, 1,4$-dioxane, $24 \mathrm{~h}$

Scheme 13 Experiments carried out to confirm carbocationic intermediates under $\mathrm{FeCl}_{3} \cdot 6 \mathrm{H}_{2} \mathrm{O}$ catalysis

We have also developed a greener strategy using this methodology; the catalyst $\mathrm{FeCl}_{3} \cdot 6 \mathrm{H}_{2} \mathrm{O}$ is used with water as the solvent. ${ }^{30}$ This protocol is the first example of the use of a Lewis acid in direct nucleophilic substitution reactions with free allylic alcohols in pure water in an $\mathrm{S}_{\mathrm{N}} 1$ type reaction. As expected, harsher conditions were necessary to reach satisfactory results; using $10 \mathrm{~mol} \%$ $\mathrm{FeCl}_{3} \cdot 6 \mathrm{H}_{2} \mathrm{O}$ at a temperature of $90{ }^{\circ} \mathrm{C}$ gave similar or slightly inferior results to those obtained in organic solvents (Scheme 14). However, it is remarkable that some carbon nucleophiles, such as TMSCN (4), which failed completely when the reaction was carried out in organic solvents, produced the desired product in water, although in a modest $45 \%$ yield. The need for the use of more forcing conditions can be easily understood from a solubility point of view, but also can be ascribed to the formation of less Lewis acidic species $\left[\mathrm{Fe}\left(\mathrm{H}_{2} \mathrm{O}\right)_{6-\mathrm{n}}(\mathrm{OH})_{\mathrm{n}}\right]^{+3-\mathrm{n}} \mathrm{Cl}_{3-\mathrm{n}} \cdot{ }^{30}$ 


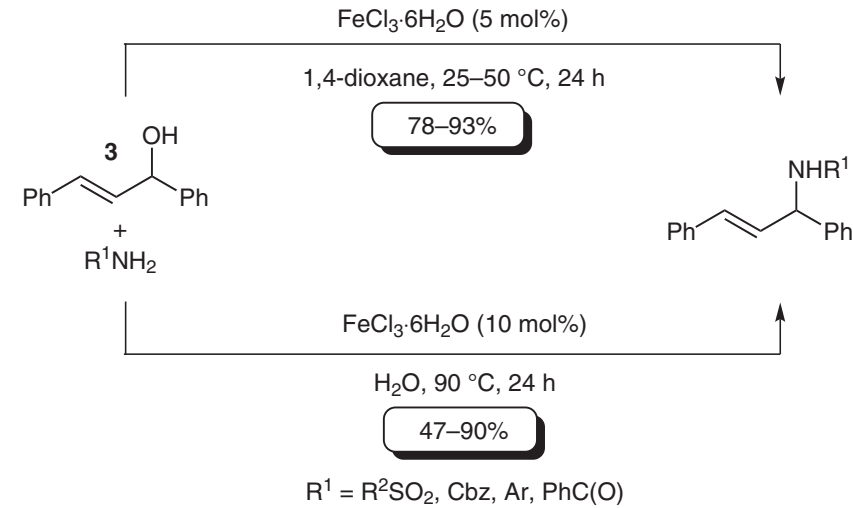

Scheme 14 Direct allylic substitution of alcohols in water catalyzed by $\mathrm{FeCl}_{3} \cdot 6 \mathrm{H}_{2} \mathrm{O}$

The Wang and Sun group used $\mathrm{FeCl}_{3} \cdot 6 \mathrm{H}_{2} \mathrm{O}$ in the synthesis of dihydroquinolines through an intramolecular direct amination (Scheme 15); high yields were obtained under mild reaction conditions and using low catalyst loadings. In addition, the obtained dihydroquinolines were readily transformed in almost quantitatively yields into the corresponding quinolines following basic treatment. ${ }^{31}$

Finally, the Kaper group used $\mathrm{ZrO}_{2}$, which to the best of our knowledge has not previously been used for this type of transformation, acting as a supported Lewis acid in a $\mathrm{SiO}_{2}-\mathrm{ZrO}_{2}$ mixed oxide structure, in the Friedel-Crafts alkylation of anisole with cinnamyl alcohol (9) [Scheme 16 , (a)]. The best results were achieved when molar ratio of $\mathrm{SiO}_{2}-\mathrm{ZrO}_{2}$ was 95:5 (named SiZr5). Of particular interested is the result obtained when $(E)$-1,3-diphenylprop-2en-1-ol (3) was reacted at $110{ }^{\circ} \mathrm{C}$ with benzyl alcohol as the solvent. The desired result was an intramolecular Friedel-Crafts reaction and subsequent transfer hydrogenation (MPV-type) of the double bond to obtain the product 10, but instead the major product was the cyclopropane derivative 11, the result of the hydrogenation of cyclopropene 12 [Scheme 16, (b)]. ${ }^{32}$

\subsection{Brønsted Acids as Catalysts}

The use of Brønsted acids (BA) as catalysts for the direct nucleophilic substitution of allylic alcohols has experienced significant growth as, despite their simplicity and availability, they were previously unexploited and they

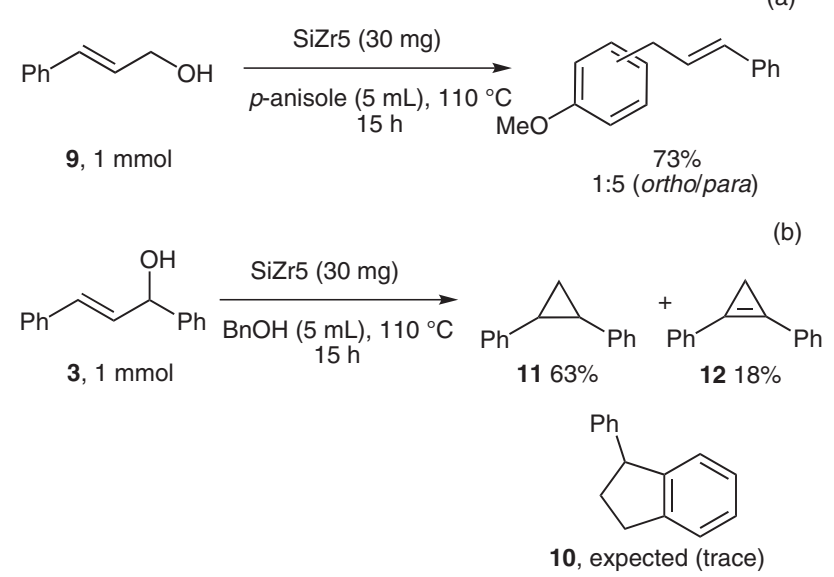

Scheme 16 Mixed oxide $\mathrm{SiO}_{2}-\mathrm{ZrO}_{2}$ catalyzed Friedel-Crafts reactions using allylic alcohols

had not been considered as potential catalysts for this transformation, with the exception of some sulfonic acids. ${ }^{5}$ The mode of action is simple, as depicted in Scheme 17. Thus, the protonation of the hydroxyl group by the Brønsted acid affords, after dehydration, the corresponding stabilized allyl carbocation that reacts with the nucleophile regenerating the Brønsted acid after proton release.

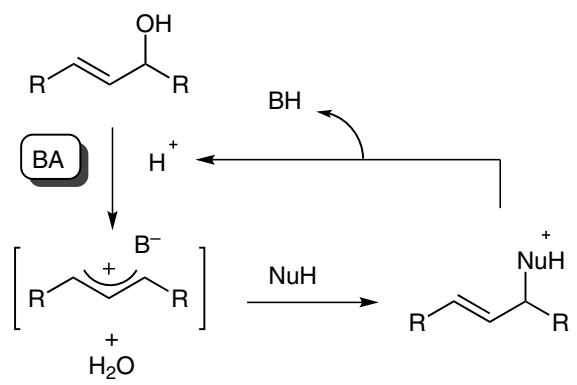

Scheme 17 Activation mode of allylic alcohols by Brønsted acids

Sulfonic acids have been the most successful Brønsted acids employed so far as catalysts for this type of transformation. This success prompted our research group, among others, to explore $\mathrm{TfOH}$ as a potential catalyst. Thus, we chose this strong acid for the direct amination of allylic alcohols in a comparative study with $\mathrm{FeCl}_{3} \cdot 6 \mathrm{H}_{2} \mathrm{O}$ as both are readily available catalysts. ${ }^{29}$ In this case, we observed

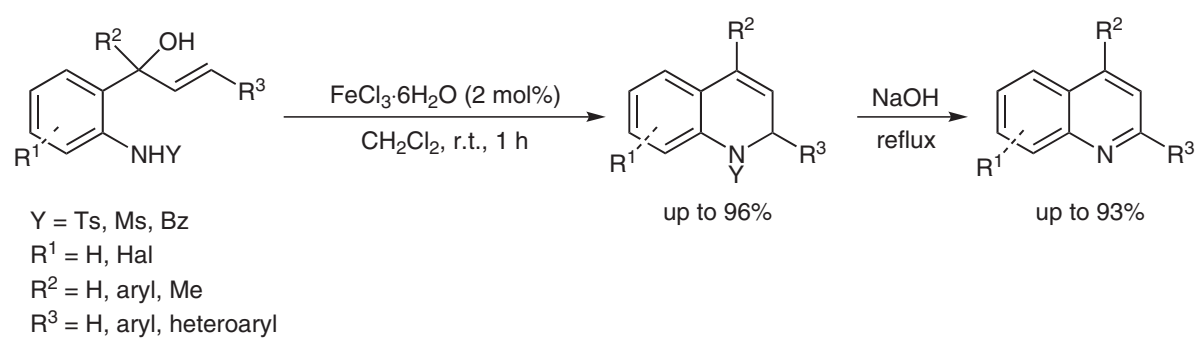

Scheme 15 Synthesis of dihydroquinolines catalyzed by $\mathrm{FeCl}_{3} \cdot 6 \mathrm{H}_{2} \mathrm{O}$ by an intramolecular amination of allylic alcohols 
that generally $\mathrm{TfOH}$ performed slightly better than the $\mathrm{Fe}(\mathrm{III})$ salt and as little as $1 \mathrm{~mol} \%$ of catalyst was sufficient to effectively promote the direct amination of a wide variety of allylic alcohols using dioxane as solvent at 25 ${ }^{\circ} \mathrm{C}$ in most cases. Remarkably, good yields (although higher temperatures were required) were achieved even when more challenging substrates, such as linear primary allylic alcohols cinnamyl alcohol (9) and (E)-but-2-en-1ol (13) were used; utilizing $\mathrm{FeCl}_{3} \cdot 6 \mathrm{H}_{2} \mathrm{O}$ with the same substrate with high temperatures and catalyst loadings gave the corresponding products in low yields (Scheme 18). However, it is important to note that the reaction with $\mathrm{TfOH}$ failed completely using water as the solvent, this is in contrast to $\mathrm{FeCl}_{3} \cdot 6 \mathrm{H}_{2} \mathrm{O}$.

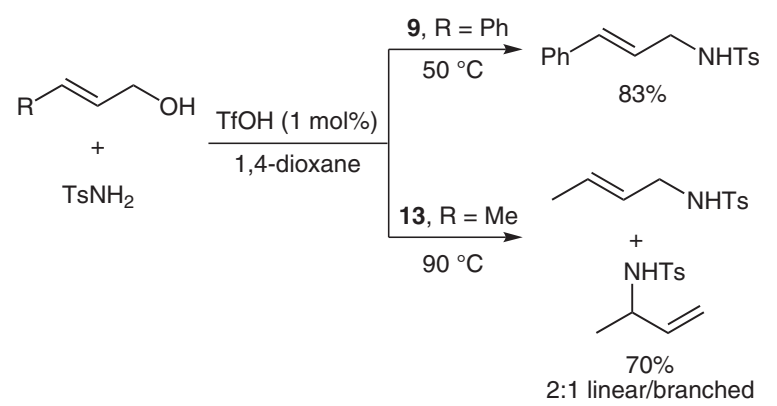

Scheme 18 Direct amination of linear primary allylic alcohols catalyzed by TfOH

This direct amination reaction has been also described using sulfonic acid functionalized ionic liquids as catalysts (Figure 2). The reaction proceeds well in terms of yield for different sulfonamides, amides, and anilines, when 10 $\mathrm{mol} \%$ of the ionic liquids was used in dioxane at $80{ }^{\circ} \mathrm{C}$ and with (E)-1,3-diphenylprop-2-en-1-ol (3) as the substrate of choice. The catalyst can be recovered by simply aqueous extraction from the reaction crude and reused up to six times without significant loss of activity. ${ }^{33}$

Jin et al. described an interesting transformation also catalyzed by $\mathrm{TfOH}$ and leading to $\mathrm{Csp}^{3}-\mathrm{Csp}^{2}$ bond formation. The reaction of allylic (and benzylic) alcohols with styrene and other alkenes (Scheme 19), gave particularly high yields for alcohol 3. ${ }^{34}$

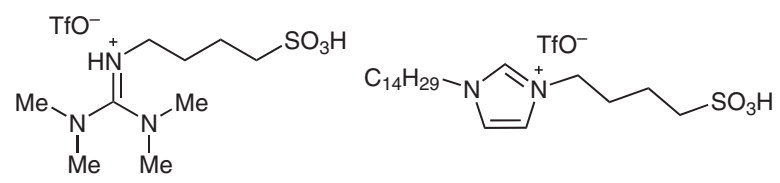

Figure 2 Sulfonic acid functionalized ionic liquids

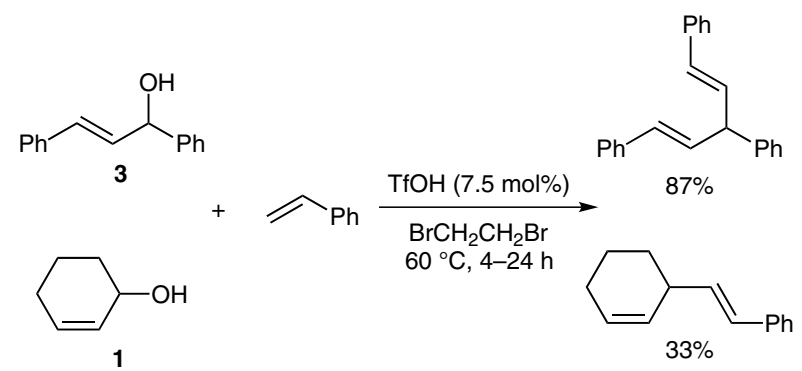

Scheme $19 \mathrm{Csp}^{3}-\mathrm{Csp}^{2}$ Bond formation catalyzed by $\mathrm{TfOH}$

Although the effectiveness of 4-toluenesulfonic acid (PTSA) as the catalyst in this type of transformation has been clearly demonstrated, especially by the Sanz group, ${ }^{35}$ remarkable work has now been published on the use of PTSA as a catalyst in $\mathrm{S}_{\mathrm{N}} 1$-type reactions (Scheme 20). Reddy et al. described a highly stereoselective C5-alkylation of oxoindoles with different alcohols. Interestingly, the alkylation occurs at C5 and not at C3 ( $\alpha-$ alkylation) as happens when Lewis acids or basic media are employed [Scheme 20, (a)]. ${ }^{36}$ In contrast, the $\alpha$-alkylation of carbonyl compounds, such as aldehydes, was obtained using PTSA under mild reaction conditions with excellent results especially for $(E)$-1,3-diphenylprop-2en-1-ol (3) [Scheme 20, (b)]. ${ }^{37,38}$

The $\alpha$-alkylation of various aldehydes using alcohol $\mathbf{3}$ has been also reported using PTSA $(20 \mathrm{~mol} \%)$ with acetonitrile as the solvent in combination with a polysiloxane (10 mol\%) bearing a secondary amine in the structure in a dual activation strategy. Thus, whereas PTSA activates the allylic alcohol forming the corresponding carbocation, the amine forms the corresponding enamine, therefore activating the aldehyde. The yields obtained were moderate

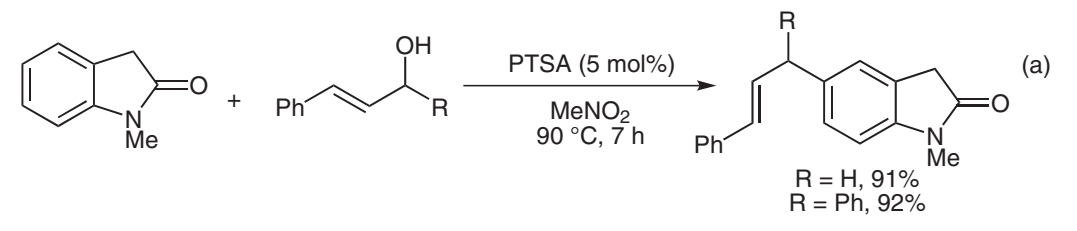<smiles>[R]C([PH3+])C(=O)c1ccccc1</smiles>

Scheme 20 Alkylation employing allylic alcohols catalyzed by PTSA 
to high; the polysiloxane can be easily separated and reused at least in 10 cycles without detriment to the results (Figure 3)..$^{39}$<smiles>C[Si](C)(C)O[Si](C)(C)O[Si](C)(C)CCCNC1CCCCC1</smiles>

Figure 3 Recyclable polysiloxane for the $\alpha$-alkylation of aldehydes

Liu and co-workers reported that other strong acids such as sulfuric ${ }^{40 a}$ and tetrafluoroboric acid ${ }^{40 b}$ effectively accomplish the direct substitution of alcohols with 1,3-dicarbonyl compounds under conventional or microwaveassisted heating. Only a few examples of allylic substitution were given using alcohol $\mathbf{3}$ as the substrate, however, the corresponding products were obtained in high yields. In both cases employed $5 \mathrm{~mol} \%$ of catalyst loading with nitromethane as the solvent.

The Aoyama group employed $\mathrm{NaHSO}_{4}$ supported on silica gel (2.1 $\mathrm{mmol} \mathrm{NaHSO}_{4} / \mathrm{g}$ silica gel) in a heterogeneous allylation of dicarbonyl and arenes compounds with free alcohols; good yields were obtained when 3 was the allylic alcohol employed. The use of less activated alcohols result in lower yields and, for example, the reaction of cinnamyl alcohol failed completely under these conditions. The reaction was generally performed at $80{ }^{\circ} \mathrm{C}$ with 1,2-dichloroethane as the solvent of choice. However, it is remarkable that the catalyst can be recycled up to nine times if it is separated from the reaction media and dried under vacuum prior to reuse. ${ }^{41}$

The McCubbin group described the use of pentafluorophenylboronic acid in intermolecular, Friedel-Craftstype, allylic substitution reactions of alcohols using different arenes and heteroarenes. ${ }^{5,42}$ From this precedent, the Hall group reported an intramolecular carbo- and heterocyclization reaction employing this catalyst (Scheme 21) to obtain a wide variety of heterocycles. ${ }^{43}$ The reaction generally affords high yields and diastereoselectivities and, according to some experiments, which were carried out in order to elucidate the mechanism, proceeds through a carbocationic intermediate rather than an $\mathrm{S}_{\mathrm{N}} 2^{\prime}$ pathway, which is the alternative suggested in other papers by the same authors ${ }^{43}$ and also by the McCubbin group. ${ }^{42}$

Finally, montmorillonites are excellent solid Brønsted acids that are able to accomplish direct nucleophilic substitution of free alcohols, ${ }^{5}$ Onaka and co-workers have reported the use of highly active $\mathrm{Sn}$ - and Ti-based montmorillonites, acting as heterogeneous Brønsted acids (although a secondary role of the metals acting as Lewis

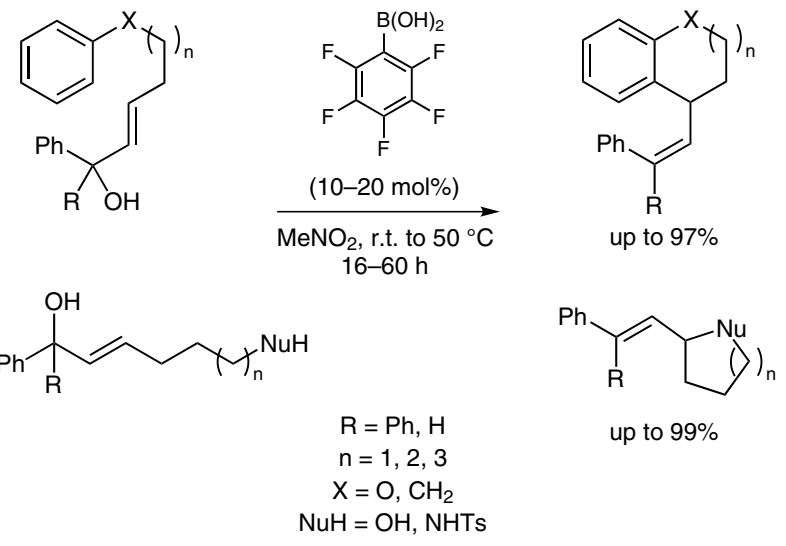

Scheme 21 Intramolecular carbo- and heterocyclization of allylic alcohols catalyzed by pentafluorophenylboronic acid

acids cannot be discounted), for the cyanation of various allylic alcohols. It is claimed that the presence of these metals enhances the Brønsted acidity of montmorillonites, since their absence, or the presence of others metals, produced poor results at best. The reaction proceeds under mild conditions (r.t.) and high yields (63-98\%) are obtained within short reaction periods (normally less than 1 h) even with low catalyst loadings ( $<3 \mathrm{~mol} \%$ ) using dichloromethane as the solvent for a wide variety of linear allylic alcohols. ${ }^{44}$

\subsection{Other Promoters}

In the search for a simple and metal-free strategy and lower acidic conditions for the direct nucleophilic substitution of allylic alcohols and, due to their unique properties, such as high hydrogen bond donor ability, high polarity, and ionizing power along with low nucleophilicity, ${ }^{45}$ we envisaged fluorinated alcohols as a reaction medium that would be able to mediate this transformation. Particularly, 2,2,2-trifluoroethanol (TFE) and 1,1,1,3,3,3-hexafluoropropan-2-ol (HFIP) were used as solvents and promoters of allylic substitution reactions of various alcohols using a wide variety of nitrogen and carbon nucleophiles (Scheme 22). ${ }^{46}$ The reaction is simple, works under mild reaction conditions (r.t. to $70{ }^{\circ} \mathrm{C}$ ), and affords the corresponding products in high yields. HFIP generally performs better than TFE, and, for example, HFIP enables the use of basic amines (such as benzylamine or butanamine) as nucleophiles with good results; these reactions generally fail under Lewis or Brønsted acid catalysis. Particularly interesting are the results obtained with rather electronrich anilines, such as 4-chloroaniline (14), which can behave as a nitrogen or carbon nucleophiles depending on the solvent employed. This was studied and it was shown to be a consequence of the reversibility of the amination reaction when HFIP was used as the solvent (Scheme 23). ${ }^{46}$ 


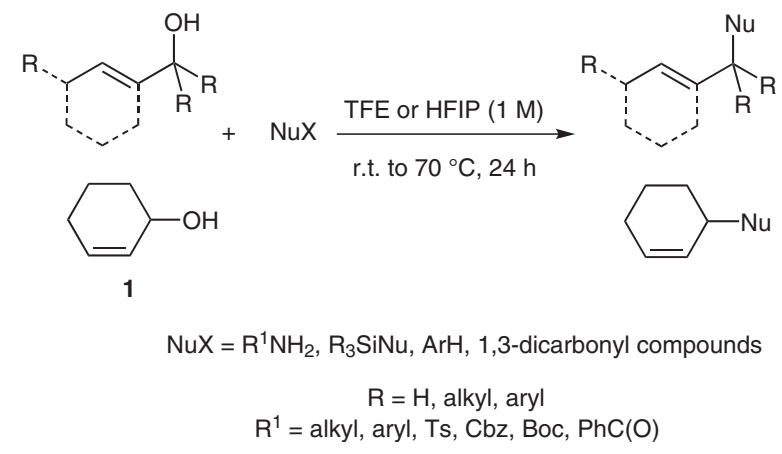

Scheme 22 Fluorinated alcohols as promoters of the direct allylic substitution of free alcohols

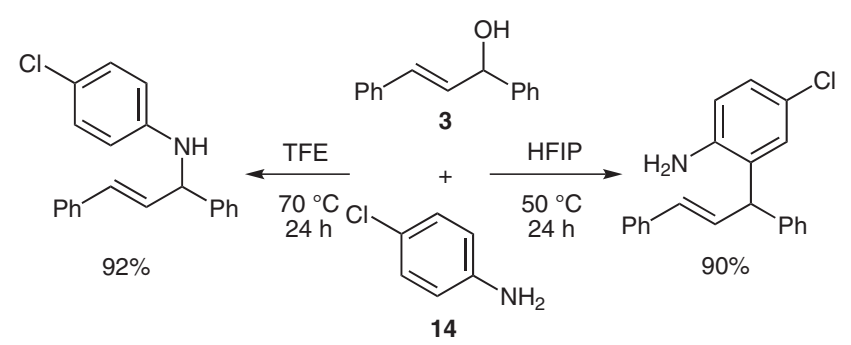

Scheme 23 Different nucleophilicity behavior of aniline 14 depending on the solvent

Studies of the elucidation of the mechanism have also been reported, confirming the existence of carbocationic intermediates, obtained through hydrogen bonding interactions, and two working reaction pathways were proposed to give the allylic substitution product: the direct amination of the alcohol-derived carbocation and the indirect allylic substitution of the carbocation derived from the corresponding fluorinated ethers. ${ }^{46}$

Another procedure, although mainly focused on benzylic alcohols, employs the solvent as promoter in a strategy for this transformation reported by Wang et al. A Zn(II)based ionic liquid, obtained mixing choline chloride and $\mathrm{ZnCl}_{2}$ in a 1:2 ratio was used as a recoverable promoter (up to 5 times) of this $\mathrm{S}_{\mathrm{N}} 1$-type allylic substitution reaction using carbon and nitrogen nucleophiles. ${ }^{47}$

\section{Conclusions and Outlook}

In conclusion, it can be asserted that great progress has been already achieved in the last decade in the $\mathrm{S}_{\mathrm{N}} 1$-type direct allylic nucleophilic substitution reactions of free alcohols and a number of methods are available to successfully perform this transformation. ${ }^{5}$ However, there is still room for improvement in various aspects of this transformation.

One point is the use of organic solvents. In this sense, efforts are now focused on the use of environmentally benign or recoverable solvents, such as water or ionic liquids.
Another field that is still undeveloped is the use of allylic alcohols in enantioselective transformations through a $\mathrm{S}_{\mathrm{N}} 1$-type reaction. ${ }^{48}$ In this regard, some advances, mainly focused on organocatalytic transformations, have been reported. However, the scope of allylic alcohols is still limited and only those that generate a highly stable carbocationic intermediate have been employed so far. Therefore, new methodologies that would allow a broader substrate scope are required.

\section{Acknowledgment}

Spanish Ministerio de Ciencia e Innovación (MICINN) (projects CTQ2010-20387 and Consolider Ingenio 2010, CSD2007-00006), FEDER, the Generalitat Valenciana (PROMETEO 2009/039) and the University of Alicante are gratefully acknowledged for financial support. A.B. would also like to thank MICINN for a Juan de la Cierva contract (JCI-2009-03710) and the University of Alicante (Project GRE12-03).

\section{References}

(1) For recent reviews about the principles of green chemistry, see: (a) Sheldon, R. A. Chem. Soc. Rev. 2012, 41, 1437. (b) Constable, D. J. C.; Dunn, P. J.; Hayler, J. D.; Humphrey, G. R.; Leazer, J. L. Jr; Linderman, R. J.; Lorenz, K.; Pearlman, B. A.; Wells, A.; Zaks, A.; Zhang, T. Y. Green Chem. 2007, 9, 411; and references therein.

(2) Trost, B. M. Angew. Chem., Int. Ed. Engl. 1995, 34, 259.

(3) For example, see: (a) Paquin, J.-F.; Lautens, M. In Comprehensive Asymmetric Catalysis, Supplement 2; Jacobsen, E. N.; Pfaltz, A.; Yamamoto, H., Eds.; Springer: Heidelberg, 2004, 73. (b) Palladium Reagents and Catalysis; Tsuji, J., Ed.; Wiley: Chichester, 2004.

(c) Handbook of Organopalladium Chemistry for Organic Systems; Vol. II; Negishi, E., Ed.; Wiley: New York, 2002, Chap. V.2, 1669. For reviews about asymmetric allylic substitutions for example, see: (d) Trost, B. M.; Zhang, T.; Sieber, J. D. Chem. Sci. 2010, 1, 427. (e) Miyabe, H.; Takemoto, Y. Synlett 2005, 1641. (f) Trost, B. M.; Crawley, M. L. Chem. Rev. 2003, 103, 2921; and references therein.

(4) For recent reviews on transition-metal-catalyzed allylic substitution reactions of alcohols, see: (a) Sundararaju, B.; Achard, M.; Bruneau, C. Chem. Soc. Rev. 2012, 41, 4467. (b) Muzart, J. Eur. J. Org. Chem. 2007, 3077.

(5) For recent review on $\mathrm{S}_{\mathrm{N}} 1$-type direct nucleophilic substitution of free alcohols, see: Emer, E.; Sinisi, R.; Guiteras-Capdevila, M.; Petruzziello, D.; De Vincentiis, F.; Cozzi, P. G. Eur. J. Org. Chem. 2011, 647.

(6) For recent reviews about the use of gold as catalyst in transformations involving alcohols, see: (a) Cera, G.; Chiarucci, M.; Bandini, M. Pure Appl. Chem. 2012, 84 , 1673. (b) Biannic, B.; Aponick, A. Eur. J. Org. Chem. 2011, 6605. (c) Muzart, J. Tetrahedron 2008, 64, 5815.

(7) Biswas, S.; Samec, J. S. M. Chem. Asian. J. 2013, 8, 974.

(8) Giner, X.; Trillo, P.; Nájera, C. J. Organomet. Chem. 2011, 696, 357.

(9) Chen, G.-Q.; Xu, Z.-J.; Cahn, S. L.-F.; Zhou, C.-Y.; Che, C.-M. Synlett 2011, 2713.

(10) Rueping, M.; Vila, C.; Uria, U. Org. Lett. 2012, 14, 768.

(11) Ren, K.; Li, P.; Wang, L.; Zhang, X. Tetrahedron 2011, 67, 2753.

(12) Our group, among others, reported the use of cationic gold(I) complexes for the direct amination reaction onto free allylic alcohols (see refs. 6 and 8). 
(13) Ohshima, T.; Nakahara, Y.; Ipposhi, J.; Miyamoto, Y.; Mashima, K. Chem. Commun. 2011, 47, 8322.

(14) Ohshima, T.; Ipposhi, J.; Nakahara, Y.; Shibuya, R.; Mashima, K. Adv. Synth. Catal. 2012, 354, 2447.

(15) Yamamoto, H.; Ho, E.; Sasaki, I.; Mitsutake, M.; Takagi, Y.; Imagawa, H.; Nishizawa, M. Eur. J. Org. Chem. 2011, 2417.

(16) Babu, S. A.; Yasuda, M.; Tsukahara, Y.; Yamauchi, T.; Wada, Y.; Baba, A. Synthesis 2008, 1717.

(17) Fan, G.-P.; Liu, Z.; Wang, G.-W. Green Chem. 2013, 15, 1659.

(18) Theerthagiri, P.; Lalitha, A. Tetrahedron Lett. 2012, 53, 5535.

(19) Meng, B.; Ma, S. Org. Lett. 2012, 14, 2674.

(20) Qin, H.; Yamagiwa, N.; Matsunaga, S.; Shibasaki, M. Angew. Chem. Int. Ed. 2007, 46, 409.

(21) (a) Narayana-Kumar, G. G. K. S.; Laali, K. K. Org. Biomol. Chem. 2012, 10, 7347. (b) Aridoss, G.; Laali, K. K. Tetrahedron Lett. 2011, 52, 6859.

(22) Shukla, P.; Choudhary, M. K.; Nayak, S. K. Synlett 2011, 1585.

(23) Das, B. G.; Nallagonda, R.; Ghorai, P. J. Org. Chem. 2012, $77,5577$.

(24) Heterobimetallic Sn-Pd complex as catalyst: (a) Das, D.; Pratihar, S.; Roy, U. K.; Mal, D.; Roy, S. Org. Biomol. Chem. 2012, 10, 4537. Heterobimetallic Sn-Ir complex as catalyst: (b) Chatterjee, P. N.; Roy, S. Tetrahedron 2012, 68, 3776. (c) Maity, A. K.; Chatterjee, P. N.; Roy, S. Tetrahedron 2013, 69, 942.

(25) (a) Meyer, V. J.; Niggemann, M. Eur. J. Org. Chem. 2011, 3671. (b) Haubenreisser, S.; Niggemann, M. Adv. Synth. Catal. 2011, 353, 469. (c) Niggemann, M.; Meel, M. J. Angew. Chem. Int. Ed. 2010, 49, 3684. For a review on the use of $\mathrm{Ca}(\mathrm{II})$ as a Lewis Acid catalyst, see: (d) Begouin, J.M.; Niggemann, M. Chem. Eur. J. 2013, 19, 8030.

(26) Liu, Z.; Wang, D.; Chen, Y. Lett. Org. Chem. 2011, 8, 73.

(27) (a) Li, M.-M.; Zhang, Q.; Yue, H.-L.; Ma, L.; Ji, J.-X. Tetrahedron Lett. 2012, 53, 317. The analogous alkenyl bromides and chlorides were previously reported using 40 $50 \mathrm{~mol} \%$ of the $\mathrm{FeBr}_{3}$ and $\mathrm{FeCl}_{3}$, respectively, see: (b) Biswas, S.; Maiti, S.; Jana, U. Eur. J. Org. Chem. 2009, 2354. (c) Liu, Z.-Q.; Wang, J.; Han, J.; Zhao, Y.; Zhou, B. Tetrahedron Lett. 2009, 50, 1240.

(28) For intermolecular version, see: (a) Jana, U.; Biswas, S.; Maiti, S. Tetrahedron Lett. 2007, 48, 4065. (b) Jana, U.; Maiti, S.; Biswas, S. Tetrahedron Lett. 2007, 48, 7160. (c) Jana, U.; Maiti, S.; Biswas, S. Tetrahedron Lett. 2008, 49, 858. For intramolecular version, see: (d) Guérinot, A.; Serra-Muns, A.; Gnamm, C.; Bensoussan, C.; Reymond, S.; Cossy, J. Org. Lett. 2010, 12, 1808. (e) Guérinot, A.; SerraMuns, A.; Bensoussan, C.; Reymond, S.; Cossy, J. Tetrahedron 2011, 67, 5024.

(29) Trillo, P.; Baeza, A.; Nájera, C. Eur. J. Org. Chem. 2012, 2929.
(30) Trillo, P.; Baeza, A.; Nájera, C. ChemCatChem 2013, 5, 1538.

(31) Wang, Z.; Li, S.; Yu, B.; Wu, H.; Wang, Y.; Sun, X. J. Org. Chem. 2012, 77, 8615.

(32) Kaper, H.; Bouchmella, K.; Mutin, P. H.; Goettmann, F. ChemCatChem 2012, 4, 1813.

(33) (a) Han, F.; Yang, L.; Li, Z.; Xia, C. Adv. Synth. Catal. 2012, 354,1052 . The same sulfonic acid functionalized ionic liquids have been successfully employed for the reaction of dicarbonyl compounds with benzylic, allylic, and propargylic alcohols: (b) Funabiki, K.; Komeda, T.; Kubota, Y.; Matsui, M. Tetrahedron 2009, 65, 7457.

(34) Yue, H.-L.; Wei, W.; Li, M.-M.; Yang, Y.-R.; Ji, J.-X. Adv. Synth. Catal. 2011, 353, 3139.

(35) For example, see: Sanz, R.; Martínez, A.; Miguel, D.; Álvarez-Gutiérrez, J. M.; Rodríguez, F. Adv. Synth. Catal. 2006, 348, 1841 .

(36) Reddy, C. J.; Jithender, E.; Krishna, G.; Reddy, G. V.; Jagadeesh, B. Org. Biomol. Chem. 2011, 9, 3940.

(37) Xing, C.; Sun, H.; Zhang, J.; Li, G.; Chi, Y.-R. Chem. Eur. J. 2011, 17, 12272.

(38) For the asymmetric version of this reaction using alcohols that give highly stabilized carbocations and catalyzed by $\mathrm{InBr}_{3}$ and imidazolidinone MacMillan's organocatalyst, see: Guiteras-Capdevila, M.; Benfatti, F.; Zoli, L.; Stenta, M.; Cozzi, P. G. Chem. Eur. J. 2010, 16, 11237.

(39) Zheng, Z.-J.; Liu, L.-X.; Gao, G.; Dong, H.; Jiang, J.-X.; Lai, G.-Q.; Xu, L.-W. RSC Adv. 2012, 2, 2895.

(40) For the use of $\mathrm{H}_{2} \mathrm{SO}_{4}$, see: (a) Xia, F.; Zhao, Z. L.; Liu, P. N. Tetrahedron Lett. 2012, 53, 2828. For the use of $\mathrm{HBF}_{4}$, see: (b) Liu, P. N.; Xia, F.; Ren, Y.; Chen, J. Chin. J. Chem. 2011, 29, 2075.

(41) Aoyama, T.; Miyota, S.; Takido, T.; Kodomari, M. Synlett 2011, 2971.

(42) For example, see: McCubbin, J. A.; Hosseini, H.; Krokhin, O. V. J. Org. Chem. 2010, 75, 959.

(43) Zheng, H.; Ghanbari, S.; Nakamura, S.; Hall, D. G. Angew. Chem. Int. Ed. 2012, 51, 6187.

(44) Wang, J.; Masui, Y.; Onaka, M. ACS Catal. 2011, 1, 446.

(45) For reviews about fluorinated alcohols in organic transformations, see: (a) Begué, J.-P.; Bonnet-Delpon, D.; Crousse, B. Synlett 2004, 18. (b) Shuklov, I. A.; Dubrovina, N. V.; Börner, A. Synthesis 2007, 2925.

(46) Trillo, P.; Baeza, A.; Nájera, C. J. Org. Chem. 2012, 77, 7344.

(47) Zhu, A.; Li, L.; Wang, J.; Zhuo, K. Green Chem. 2011, 13, 1244.

(48) For reviews on the use of allylic alcohols in asymmetric catalysis, see: (a) Bandini, M. Angew. Chem. Int. Ed. 2011, 50, 994. (b) Bandini, M.; Cera, G.; Chiarucci, M. Synthesis 2012, 44, 504. 